\title{
Auditory ERP response to successive stimuli in infancy
}

Ao Chen, Varghese Peter, Denis Burnham

Background. Auditory event-related potentials (ERPs) are useful for understanding early auditory development among infants, as it allows the collection of a relatively large amount of data in a short time. So far, studies that have investigated development in auditory ERPs in infancy have mainly used single sounds as stimuli. Yet in real life, infants must decode successive rather than single acoustic events. In the present study, we tested 4-, 8-, and 12-month-old infants' auditory ERPs to musical melodies comprising three piano notes, and examined ERPs to each individual note in the melody.

Methods. Infants were presented with 360 repetitions of a three-note melody while EEG was recorded from 128 channels on the scalp through a Geodesic Sensor Net. For each infant both latency and amplitude of auditory components P1 and N2 were measured from averaged ERPs for each individual note.

Results. Analysis was restricted to response collected at frontal central site. For all three notes there was an overall reduction in latency for both P1 and N2 over age. For P1, latency reduction was significant from 4 to 8 months, but not from 8 to 12 months. N2 latency, on the other hand, decreased significantly from 4 to 8 to 12 months. With regard to amplitude, no significant change was found for either P1 or N2. Nevertheless, the waveforms of the three age groups were qualitatively different: for the 4-month-olds, the P1-N2 deflection was attenuated for the second and the third notes; for the 8-month-olds, such attenuation was observed only for the middle note; for the 12-month-olds, the P1 and N2 peaks show relatively equivalent amplitude and peak width across all three notes. Conclusion. Our findings indicate that the infant brain is able to register successive acoustic events in a stream, and ERPs become better time-locked to each composite event over age. Younger infants may have difficulties in responding to late occurring events in a stream, and the onset response to the late events may overlap with the incomplete response to preceding events. 
Auditory ERP Response to Successive Stimuli in Infancy Ao Chen ${ }^{1,2,3}$, Varghese Peter $^{1}$, \& Denis Burnham ${ }^{1}$

1. MARCS Institute, Western Sydney University, Australia

2. Utrecht Institute of Linguistics, Utrecht University, the Netherlands

3. Department of Psychiatry, Brain Center Rudolf Magnus, University Medical Center Utrecht, the Netherlands
Corresponding author: Ao Chen, irischen71@hotmail.com, Trans 10, 3512JK, Utrecht, the Netherlands. Tel: $+31-30-253-6407$ 


\section{Abstract}

24 Background. Auditory event-related potentials (ERPs) are useful for understanding early auditory development among infants, as it allows the collection of a relatively large amount of data in a short time. So far, studies that have investigated development in auditory ERPs in

27 infancy have mainly used single sounds as stimuli. Yet in real life, infants must decode

28 successive rather than single acoustic events. In the present study, we tested 4-, 8-, and 12-

29 month-old infants' auditory ERPs to musical melodies comprising three piano notes, and

30 examined ERPs to each individual note in the melody.

31 Methods. Infants were presented with 360 repetitions of a three-note melody while EEG was

32 recorded from 128 channels on the scalp through a Geodesic Sensor Net. For each infant both

33 latency and amplitude of auditory components P1 and N2 were measured from averaged ERPs

34 for each individual note.

35 Results. Analysis was restricted to response collected at frontal central site. For all three notes

36 there was an overall reduction in latency for both $\mathrm{P} 1$ and $\mathrm{N} 2$ over age. For P1, latency reduction

37 was significant from 4 to 8 months, but not from 8 to 12 months. N2 latency, on the other hand,

38 decreased significantly from 4 to 8 to 12 months. With regard to amplitude, no significant

39 change was found for either P1 or N2. Nevertheless, the waveforms of the three age groups were

40 qualitatively different: for the 4-month-olds, the P1-N2 deflection was attenuated for the second 
41 and the third notes; for the 8-month-olds, such attenuation was observed only for the middle note;

42 for the 12-month-olds, the P1 and N2 peaks show relatively equivalent amplitude and peak width

43 across all three notes.

44 Conclusion. Our findings indicate that the infant brain is able to register successive acoustic

45 events in a stream, and ERPs become better time-locked to each composite event over age.

46 Younger infants may have difficulties in responding to late occurring events in a stream, and the

47 onset response to the late events may overlap with the incomplete response to preceding events.

48

49

50

51

52

53

54

55

56

57

58

59

60 


\section{Introduction}

Electrical responses in the brain to external or internal events are called event-related

potentials (ERPs). While various behavioural methods are available, ERPs are especially useful

67 for studying auditory development early in infancy. Behavioral methods measure infants' auditory perception indirectly, using for example, looking time as a proxy for auditory attention, but as active attention is required, such methods are limited by infants' attentional capacity and so typically only relatively small amounts of data can be collected from each infant. In contrast, auditory ERP data collection does not require active participation, and a fairly large amount of data can be obtained within a short time period, which makes it a useful tool for studying auditory development in the early years of life (Dehaene-Lambertz \& Baillet, 1998; He, Hotson, \& Trainor, 2007; Kushnerenko, Ceponiene, Balan, Fellman, \& Näätänen, 2002; Trainor, 2012) Auditory ERPs mature from birth to childhood. The usual peaks in adult auditory ERPs, P1 (positive deflection between 40-60 ms after stimulus onset), N1 (negative deflection between 90-110 ms after stimulus onset), P2 (positive deflection between 140-170 ms after stimulus onset), N2 (negative deflection between 220-280 ms after stimulus onset), may not be visible in infant auditory ERPs (Barnet, Ohlrich, Weiss, \& Shanks, 1975; Ceponiene, Cheour, \& Näätänen, 1998; Kushnerenko et al., 2002; Näätänen, 1992; Ponton, Eggermont, Kwong, \& Don, 2000; 
81 Wunderlich, Cone-Wesson, \& Shepherd, 2006). When presented with stimuli at inter-stimulus

82 intervals (ISIs) shorter than $1000 \mathrm{~ms}$, the auditory ERPs of infants and young children exhibit a

83 positive peak around 150ms (P1) after stimulus onset, a negative peak around 250ms (N2),

84 another positive peak at around 350ms (P2), and another negative peak at around 450ms (N3)

85 (Ceponiene et al., 1998; Kushnerenko et al., 2002). Many studies have found decreases in

86 response latency as infants mature (Barnet, Ohlrich, Weiss, \& Shanks, 1975; Ceponiene, Cheour,

87 \& Näätänen, 1998; Kushnerenko et al., 2002; Näätänen, 1992; Ponton, Eggermont, Kwong, \&

88 Don, 2000; Wunderlich, Cone-Wesson, \& Shepherd, 2006), probably due to increasing neuronal

89 myelination over age (Eggermont \& Salamy, 1988; Moore \& Guan, 2001). Yet, such reductions

90 of latency are not found for all peaks. Little et al. (1999) tested 5- to 17-week-old infants with

91 100ms duration tones as well as clicks, and found latency decrease over age for the late peaks

92 (N2, P2), but not for the earlier P1 (peak around 150ms after the stimuli onset). However, testing

93 older infants longitudinally at 3, 6, 9, and 12 months on three $100 \mathrm{~ms}$ harmonic tones,

94 Kushnerenko et al. (2002) found that P1 latency decreased as the infant grew older, whereas N2

95 latency did not show significant difference across ages. The different patterns in these two

96 studies suggest a non-linear development of peak latency, i.e., the latency decrease for certain

97 peaks might be more evident within a specific age window. In addition, Little et al. used only

98 one tone $(400 \mathrm{~Hz})$ whereas Kushnerenko et al. (2002) used three tones $(500 \mathrm{~Hz}, 625 \mathrm{~Hz}$, and

$99750 \mathrm{~Hz}$ ) and the ERPs were obtained by averaging the responses to all the tones. These different

100 stimuli may have influenced developmental patterns. 
102 complex picture. Barnet et al. (1975) tested infants from 10 days to 37 months, using clicks as

103 stimuli, and found that P1-N2 deflection was the "landmark" to measure auditory ERP, as it is

104 present in all participants, and the P1-N2 amplitude increased over age. In the Little et al. (1999)

105 study stimulus specific developments of P1 and N2 amplitudes was found: P1 showed a

106 significant increase for clicks but not tones; N2 showed a quadratic trend for both the tones and

107 clicks, but in the opposite direction. For the tones, N2 amplitude first increased and then

108 decreased from 5 to 17 weeks, whereas the opposite was true for the N2 of the clicks.

109 Kushnerenko et al. (2002) demonstrated that P1 amplitude at birth was significantly smaller than

110 at older ages, and remained stable from 3 months to 12 months, and that $\mathrm{N} 2$ increased in

111 amplitude (became more negative) between 3 and 9 months. Jing and Benasich (2006) found that

$112 \mathrm{~N} 2$ and P3 increased between 3 and 9 months, and then decreased with age. Together these

113 findings suggest that the development of auditory ERPs is highly stimulus specific. Similar to

114 latency, amplitude change might be more evident within a specific age window for certain peaks.

115 Nevertheless, the P1-N2 deflection seems to be a reliable marker for the auditory onset response.

116 In these studies, quite often single sounds (e.g., clicks or single tones) have been used as

117 stimuli. Yet in real life, we often need to decode successive rather than single acoustic events.

118 For example, speech consists of multi-word utterances (e.g., van de Weijer, 1998), and the

119 segmentation of words from the continuous speech stream is fundamental to infants' language

120 learning. Similarly, music is composed of multi-note bars and phrases, often without pauses 
121 between notes (Krumhansl \& Jusczyk, 1990). Efficient on-line processing of real life auditory

122 input requires efficient accurate processing of these rapidly presented successive signals.

123 Impairments in such successive processing are associated with reading and language

124 impairments (Tallal, 2004) and are predictive of later language skills when assessed in infancy

125 (Choudhury \& Benasich, 2011). Therefore understanding how the infant's brain responds to

126 successive stimuli is fundamental to studying high level processing. Mismatched negativity

127 (MMN), the neural detection of regularity violation (see Näätänen, Paavilainen, Rinne, \& Alho,

128 2007; Paavilainen, 2013), is a method that has been widely used to understand human auditory

129 discrimination. An MMN is elicited when listeners encounter infrequent sounds embedded in

130 repeating frequent sounds. Multi-tone melodies have been used as stimuli to understand infants'

131 mismatched responses (Stefanics et al., 2009; Tew, Fujioka, He, \& Trainor, 2009), yet these

132 studies often incorporate relatively long inter-tone intervals between the notes composing the

133 melodies. For example, the tones used by Stefanics et al. (2009) were of extremely short duration

134 (50 ms), with a relatively long inter-tone intervals (150ms) and inter-pair intervals much longer

135 than the tones themselves $(1250 \mathrm{~ms})$.

136 In real life, however, sounds tend to have a much longer duration and occur successively

137 with much shorter inter-sound intervals, if at all. Speech syllables in running speech tend to have

138 a duration of around 150-200 ms (Koopmans-van Beinum \& van Donzel, 1996), while musical

139 tempi tend to cluster between 90 and 120 beats per minute, i.e., each beat is longer than $500 \mathrm{~ms}$,

140 and often contains multiple notes (Moelants, 2002). Previous findings have shown that infants 
141 and adults respond to fast versus slow temporal modulations differently (Telkemeyer et al., 2009;

142 Giraud \& Poeppel, 2012), hence it is important to investigate how infants' brain responds to

143 sound streams that represent real life characteristics, as this provides the basis for understanding

144 higher level neural function. Given that ERPs develop in a stimulus-specific manner (Little et al.,

145 1999; Wunderlich, Cone-Wessen, \& Shepard, 2006), it is important to gain insight into how the

146 infant brain registers real life successive acoustic events, so that higher level processing such as

147 MMN can be better understood. In the current study, we used a melody comprised of three

148 successive piano notes, and examined the development of auditory ERPs, specifically the

149 infantile auditory onset responses, the P1 and N2. We tested 4-, 8-, and 12-month-old infants,

150 and investigated how P1 and N2 latency and amplitude change across the three tones, and how

$151 \mathrm{P} 1$ and N2 to successive tones develop in the first year of life. Auditory ERPs reflect neural

152 firing time-locked to the stimuli, and exhibit high temporal resolution. As the P1-N2 onset

153 complex is generated with a longer latency among young infants, we suggest that that upon

154 encountering successive acoustic events, young infants may not be able to process each event

155 sufficiently before the next one is initiated. Older infants, on the other hand, may be able to

156 register the sounds much more rapidly, and hence may be more capable of processing each

157 successive tone in the 3-tone stimulus.

\section{Methods}

159 Ethics 
161 experimental methods used in the study (Approval number: H9660). Informed consent was

162 obtained from parents of all the participants.

\section{Participants}

164 Eighteen 4-month-old (range: 4 month 4 days-5 month 5 days, 10 girls), 18 8-month-old

165 (range: 7 month 22 days-9 month 4 days, 10 girls), and 18 12-month-old (range: 11 month 10

166 days-13 month 3 days, 11 girls) healthy infants participated in the study. All the infants were

167 raised in a monolingual or predominantly Australian English environment. The current study is

168 part of larger project, where we compare the perceptual development of musical versus speech

169 stimuli. We selected these three age groups because the infants are assumed to tune into the

170 sound structure of their native language between 4 and 12 months. None of the parents reported

171 any hearing impairment or any ear infection within the two weeks before the experiment. One

172 12-month-old girl was tested but excluded from the analysis as she pulled the EEG cap off

173 shortly after the experiment started, and one 8-month-old boy was excluded due to fussiness.

\section{Stimuli}

176 Nyquist, where the frequency of middle A (A4) was the usual $440 \mathrm{~Hz}$. The notes were generated

177 with the default duration of one- $16^{\text {th }}$ of a note $(250 \mathrm{~ms})$. Then they were concatenated to form a

178 rising melody. In order to ensure continuity and naturalness, the whole melody was then adjusted

179 in Praat (Boersma \& Weenink, 2013) using the overlap-add method to a duration of 667.5ms, 
180 which resulted in slightly different durations for each note - first note duration $=220 \mathrm{~ms}$, second

181 note duration $=227 \mathrm{~ms}$, and third note duration $=221 \mathrm{~ms}$. The stimuli were presented through

182 two audio speakers (Edirol MA-10A) each $1 \mathrm{~m}$ from the infants, and each $30 \mathrm{~cm}$ from the midline

183 of the infant's sagittal plane. The stimuli were presented at 75dB SPL, with a random inter-

184 stimulus interval varying between 450 and 550ms. The stimuli were played through Presentation

18514.9 (Neurobehavioral Systems).

186 Procedure

187 The infants sat on the caregiver's lap in a sound attenuated room. An infant friendly

188 movie was played silently on a screen $\sim 1 \mathrm{~m}$ from the infants (in between the two audio speakers)

189 to keep them engaged. Parents were instructed not to talk during the experiment. A maximum of

190360 trials were presented, but the experiment terminated if the infant became restless. The total

191 duration of the recording was around 7 minutes. EEG was recorded from 128 channels on the

192 scalp through a Geodesic Sensor Net (net sizes: 40-42cm, 43-44cm, or 44-47cm, depending on

193 the head size of the infants), and all electrodes had impedance lower than $50 \mathrm{k} \Omega$ at the beginning

194 of the experiment. The EEG was recorded at a sampling rate of $1000 \mathrm{~Hz}$, and the online recording

195 was referred to the vertex.

196 EEG analysis

197 The EEG was analyzed offline using NetStation 4.5.7 software and EEGLAB toolbox

198 (version 13.1.1b) in Matlab 2011b. The raw recordings were filtered with EGI software between

$199 \quad 0.3-20 \mathrm{hz}$. The filtered recordings were down-sampled to $250 \mathrm{~Hz}$ before further analysis. The 
200 continuous recordings were segmented into $1000 \mathrm{~ms}$ epochs from the $100 \mathrm{~ms}$ before the onset

201 (baseline) to $900 \mathrm{~ms}$ after the onset of the first note. The 15 electrodes at the peripheral positions

202 of the net were removed from the analysis due to contamination from muscle movements. Next,

203 for each participant, bad channels were identified by visual inspection and were interpolated

204 (mean number of interpolated channels $=4, \mathrm{SD}=2$ ). EEG was then re-referenced to an average

205 reference. As it was impossible for the infants to sit still during the entire experiment, ERPs

206 recorded from the parietal and occipital electrodes were contaminated by head movements.

207 Auditory ERPs are mainly central-frontally distributed, hence conducting artifact reduction on all

208 channels would waste clean signal from the central-frontal site. In order to sufficiently remove

209 artifacts while retaining sufficient data from each child, we conducted artifact rejection on the 25

210 frontal channels (Figure 1), where trials having an amplitude larger than 150 microvolts were

211 removed. The remaining artifact free trials were averaged to obtain the ERP values for each

212 infant. After artifact removal, the 4-month-olds had a mean of 248 trials (standard deviation (SD)

$213=39.87)$ accepted, the 8-month-olds a mean of 228 trials $(\mathrm{SD}=63.64)$ accepted, and the 12-

214 month-olds a mean of 275 trials $(\mathrm{SD}=38.1)$ accepted. Channel 5, 11 and 12 were averaged to

215 represent frontal central scalp ERP responses (FC), which correspond to the location of Fz on a

$21610-20$ system. Figure 1 indicates the 25 channels used for artifact reduction and the channels

217 averaged to represent FC. For each age, a grand average ERP was computed by averaging the

218 ERPs of all participants. 
220 and 500-700ms respectively from the onset of the melody. The P1 was defined as the highest

221 positive peak in the above time windows. The N2s to each note were detected in the windows

222 200-300ms, 400-600ms, and 600-700ms respectively from the onset of the melody. N2 was the

223 lowest negative peak in the above time windows. Next, for each individual participant, the P1

224 and N2 peaks for each note were identified in the $\pm 25 \mathrm{~ms}$ window of the corresponding grand

225 average peaks.

226

\section{Channel locations}

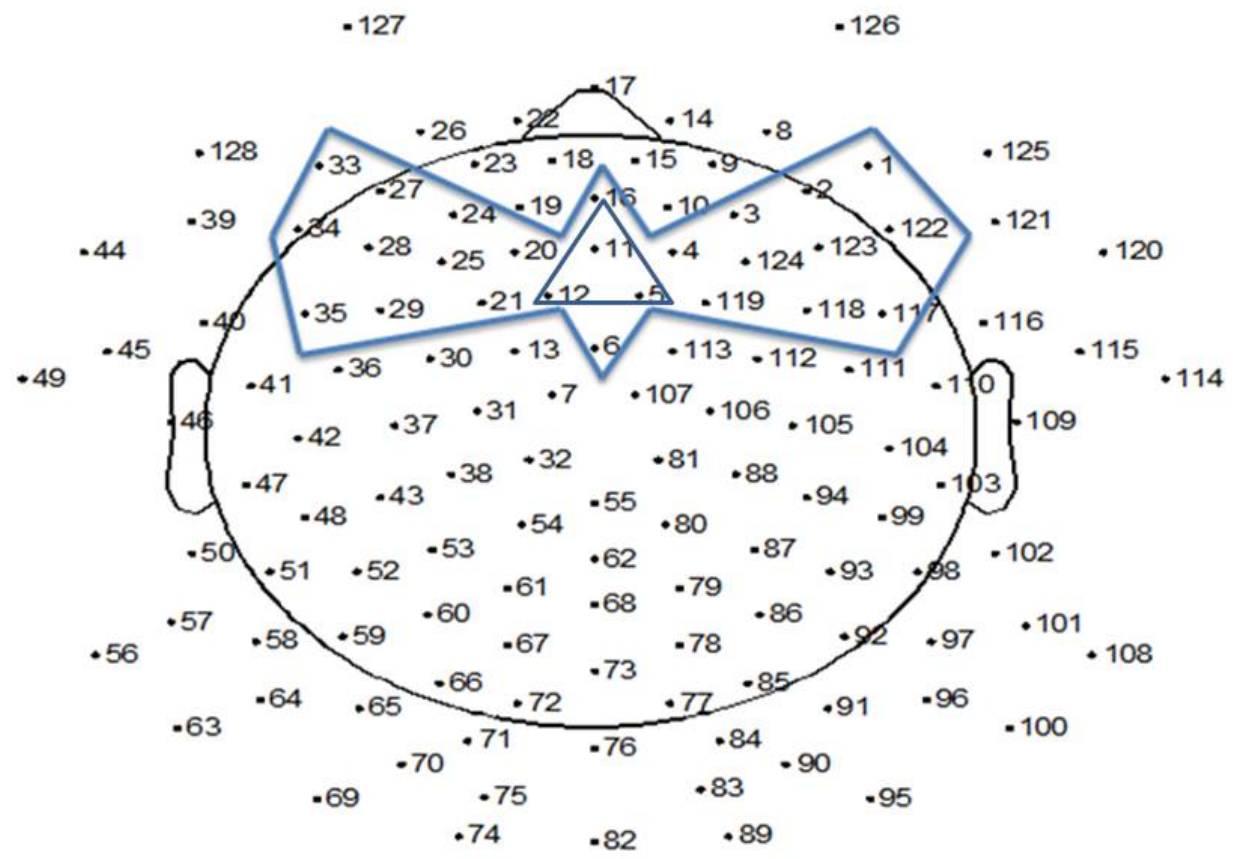


228 Figure 1 . The 25 channels used for artifact reduction (the area circumscribed by the bow-tie

229 shape) and the three channels averaged for representing response at FC (circumscribed by the

230 triangle shape).

231 Results

232 Figure 2 plots the grand average ERPs for the three age groups across time. As can be seen, the

233 onset responses for each note, namely the infantile P1 and N2, were easily identifiable at each

234 age.

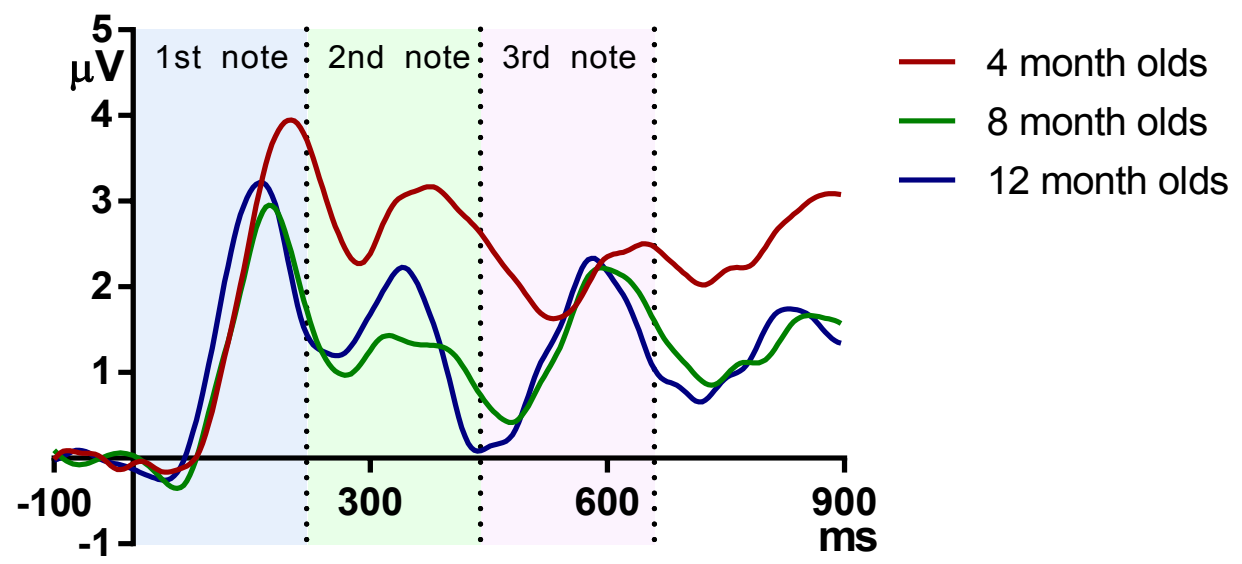

235

236 Figure 2. Grand average responses of the three age groups. The vertical axis indicates the onset

237 of the melody, and the vertical dotted lines indicate the onsets of the second and the third note.

Table 1 shows the latency of P1 and N2 for each note at FC. For each note separately, the latency measurements of P1 and $\mathrm{N} 2$ at FC were submitted to a univariate ANOVA, with age as the between-subjects variable. When age showed a significant result, Bonferroni-corrected post-

241 hoc tests were conducted for pair-wise comparisons. Table 2 shows the ANOVA results for the 242 latency measurements. 


\begin{tabular}{|l|l|l|l|}
\hline ERP peaks & $1^{\text {st }}$ note & $2^{\text {nd }}$ note & $3^{\text {rd }}$ note \\
\hline $4 \mathrm{~m} \mathrm{P1}$ & $195.78(16.49)$ & $375.55(20.86)$ & $648.89(18.59)$ \\
$4 \mathrm{~m} \mathrm{~N} 2$ & $288.44(17.35)$ & $532.89(19.43)$ & $722.89(19.30)$ \\
\hline $8 \mathrm{~m} \mathrm{P} 1$ & $169.41(13.34)$ & $325.88(20.84)$ & $587.76(17.00)$ \\
\hline $12 \mathrm{~m} \mathrm{~N} 2$ & $268.94(20.76)$ & $470.12(18.72)$ & $732.00(17.83)$ \\
\hline $12 \mathrm{~m} \mathrm{~N} 1$ & $162.12(16.68)$ & $339.53(16.36)$ & $581.65(12.50)$ \\
& $250.12(19.60)$ & $439.76(19.60)$ & $712.14(17.41)$ \\
\hline
\end{tabular}

243 Table 1. Mean P1 and N2 latency of each note with regard to the onset of the melody separated

244 by age groups. Standard deviations are given in parentheses.

\begin{tabular}{|c|c|c|}
\hline & Effect of age & Post-hoc test \\
\hline $1^{\text {st }}$ note $\mathrm{P} 1$ & $\mathrm{~F}(2,49)=22.72, \mathrm{p}<0.01$ & $\begin{array}{l}4 \mathrm{~m} \text { v.s. } 8 \mathrm{~m}, \mathrm{P}<0.01 \\
4 \mathrm{~m} \text { v.s. } 12 \mathrm{~m}, \mathrm{p}<0.01 \\
8 \mathrm{~m} \text { v.s. } 12 \mathrm{~m}, \text { n.s. }\end{array}$ \\
\hline $1^{\text {st }}$ note N2 & $\mathrm{F}(2,49)=17.33, \mathrm{p}<0.01$ & $\begin{array}{l}4 \mathrm{~m} \text { v.s. } 8 \mathrm{~m}, \mathrm{p}<0.05 \\
4 \mathrm{~m} \text { v.s. } 12 \mathrm{~m}, \mathrm{p}<0.01 \\
8 \mathrm{~m} \text { v.s. } 12 \mathrm{~m}, \mathrm{p}<0.05\end{array}$ \\
\hline $2^{\text {nd }}$ note $\mathrm{P} 1$ & $\mathrm{~F}(2,49)=30.2, \mathrm{p}<0.01$ & $\begin{array}{l}4 \mathrm{~m} \text { v.s. } 8 \mathrm{~m}, \mathrm{p}<0.01 \\
4 \mathrm{~m} \text { v.s. } 12 \mathrm{~m}, \mathrm{p}<0.01 \\
8 \mathrm{~m} \text { v.s. } 12 \mathrm{~m}, \text { n.s. }\end{array}$ \\
\hline $2^{\text {nd }}$ note $\mathrm{N} 2$ & $\mathrm{~F}(2,49)=117.92, \mathrm{p}<0.01$ & $\begin{array}{l}4 \mathrm{~m} \text { v.s. } 8 \mathrm{~m}, \mathrm{p}<0.01 \\
4 \mathrm{~m} \text { v.s. } 12 \mathrm{~m}, \mathrm{p}<0.01 \\
8 \mathrm{~m} \text { v.s. } 12 \mathrm{~m}, \mathrm{p}<0.01\end{array}$ \\
\hline
\end{tabular}




\begin{tabular}{|l|l|l|l|}
\cline { 3 - 4 } 245 & $3^{\text {rd }}$ note $\mathrm{P} 1$ & $\mathrm{~F}(2,49)=92.08, \mathrm{p}<0.01$ & $4 \mathrm{~m}$ v.s. $8 \mathrm{~m}, \mathrm{p}<0.01$ \\
246 & & $4 \mathrm{~m}$ v.s. $12 \mathrm{~m}, \mathrm{p}<0.01$ \\
247 & & $8 \mathrm{~m}$ v.s. $12 \mathrm{~m}, \mathrm{n} . \mathrm{s}$. \\
\cline { 3 - 4 } 248 & $3^{\text {rd } \text { note } \mathrm{N} 2}$ & $\mathrm{~F}(2,49)=5.01, \mathrm{p}<0.05$ & $4 \mathrm{~m}$ v.s. $8 \mathrm{~m}, \mathrm{n} . \mathrm{s}$. \\
249 & & $4 \mathrm{~m}$ v.s. $12 \mathrm{~m}, \mathrm{n} . \mathrm{s}$. \\
& & & $8 \mathrm{~m}$ v.s. $12 \mathrm{~m}, \mathrm{p}<0.01$ \\
\hline
\end{tabular}

250

251

252

253

254

255

256

257

258

259

260

261

262 Table 2. Effects of age for P1 and N2 latency measurements for each note.

264 infants. More specifically, reduction of P1 latency is mainly observed between 4 and 8 months, 
265 whereas the latency of N2 decreases monotonically and consistently from 4 to 8 to 12 months. It

266 can be observed that at the onsets of the second and the third note, the 12-month-olds had a more

267 negative deflection than the 4- and 8-month-olds, which suggests that the younger groups have a

268 later complement of N2 than the older ones.

269 Next we examined the P1 and N2 latency with regard to the onset of each note. Figure 3

270 plots the P1 and N2 latency respectively with regard to note onset for each age group. Mixed

271 design ANOVAs were conducted for P1 and N2 latencies with notes (Note1, Note2, Note3) as

272 the within-subjects variable, and age as between-subjects variable. For P1, there was an overall

273 significant main effect of note, $F(2,98)=122.49$, $p<0.01$. Bonferroni-corrected post-hoc

274 analysis indicated that P1 was significantly later for Note 1 than for Notes 2 and 3 , both $\mathrm{p}<0.001$;

275 and P1 of Note 2 was significantly earlier than both Note 1 and 3 , both $p<0.001$. Age also

276 showed a significant main effect, $F(2,49)=100.48, \mathrm{p}<0.01$. The 4-month-olds had a

277 significantly later P1 compared to the 8- and 12-month-olds $(\mathrm{p}<0.001)$ with no significant

278 difference between the 8- and 12-month-olds. A significant interaction was found between notes

279 and age, $\mathrm{F}(2,98)=8.96, \mathrm{p}<0.01$. Bonferroni post-hoc tests indicated that: for the 4-month-olds,

280 P1 of Note 2 was significantly earlier than that of Notes 1 and 3 (both $p<0.01$ ) with no

281 significant difference between Notes 1 and 3; for the 8- and 12-month-olds, P1 of Note 2 was

282 significantly earlier than that of Notes 1 and 3 (both $\mathrm{p}<0.01$ ), and P1 for Note 1 was significantly

283 later than that of Note 3 (both $\mathrm{p}<0.05$ ). 
285 Bonferroni-corrected post-hoc tests indicated that the N2 of Note 2 was significantly earlier than

286 that of Note 1 and 3, with no significant difference between these two. Age was also significant,

$287 \mathrm{~F}(2,49)=58.83, \mathrm{p}<0.01$. Bonferroni-corrected post-hoc tests indicated that the N2 latency

288 decreased significantly from 4 to 8 months, and from 8 to 12 months, both $p<0.05$. A significant

289 interaction was found between notes and age, $\mathrm{F}(2,98)=34.00, \mathrm{p}<0.01$, Bonferroni corrected

290 post-hoc tests indicated that: for 4-month-olds, N2 latency of Note 2 was significantly later than

291 that of Notes 1 and 3 (both $\mathrm{p}<0.01$ ); whereas on the other hand, for the 8- and 12-month-olds, N2

292 latency of Note 2 was significantly earlier than that of Notes 1 and 3, with N2 latency of Note 3

293 being significantly later than that of Note $1($ all $\mathrm{p}<0.05)$.
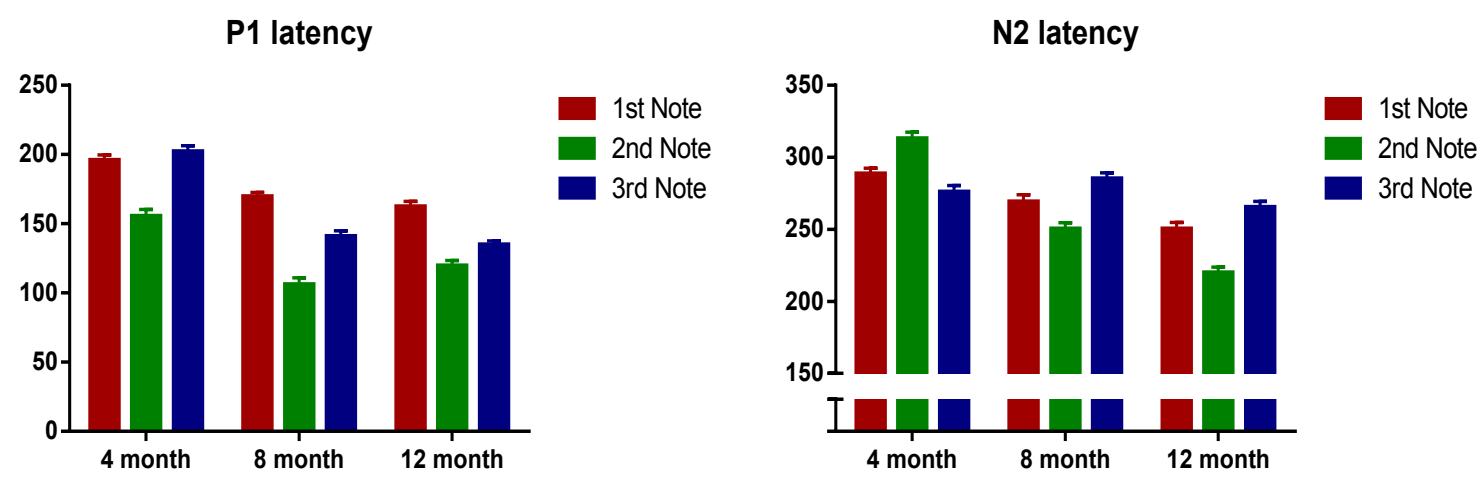

294

295 Figure 3. Mean P1 and N2 latency with regard to each note's onset of the three age groups. Error

bars represent standard errors.

In summary, P1 latency for each note decreases as the infants grow older. Interestingly,

298 for all the three ages, the P1 of the middle note, Note 2, had the shortest latency compared to P1 
299 of the edge notes. For the 4-month-olds, the P1 latency of Note 3 is longer than that of Note 1,

300 whereas the 8-and 12-month-olds showed the opposite pattern. For the N2, the 4-month-olds had

301 a longer N2 latency for Note 2, whereas for the other two groups, N2 latency was the shortest for

302 Note 2.

Mean P1 and N2 amplitudes of each note for the three age groups are provided in Table 3

304 and Figure 4 plots the mean P1 and N2 amplitude of each note of the three age groups. ANOVAs

305 were conducted for the absolute amplitude of P1 and N2 with notes as the within-subjects

306 variable and age as the between-subjects variable. For P1, a significant main effect of notes was

307 found, $\mathrm{F}(2,98)=9.01, \mathrm{p}<0.01$, but no significant main effect of age. Bonferroni-corrected pair-

308 wise analyses indicated that P1 amplitude was significant larger at Note 1, compared to that at

309 Notes 2 and 3 (both $\mathrm{p}<0.01$ ), with no difference between these two. For N2, notes had a

310 marginally significant effect, $\mathrm{F}(2,98)=2.95, \mathrm{p}=0.057$, and no significant difference was found

311 between any pair of the three notes, and there was no significant main effect of age. For neither

312 P1 nor N2, was there a significant interaction between age and notes. Similarly, for P1-N2 peak-

313 to-peak amplitude, notes again showed a significant main effect, $\mathrm{F}(2,98)=3.66$, $\mathrm{p}<0.01$; the $\mathrm{P} 1$ -

314 N2 peak-to-peak amplitude of Note 3was significantly smaller than that of Note $1(\mathrm{p}<0.05)$,

315 whereas no significant difference was found between either Note 1 and 2, or Note 2 and 3. As

316 notes had a significant main effect for all the three amplitude measurements, we examined the

317 effect of age for each note separately. For both the P1 and N2 amplitudes, age did not show a

318 significant effect for any of the notes, and no pair-wise difference was significant. For P1-N2 
319 amplitude, no significant effect of age was found for either Note 1 or 2 , but for Note 3 , there was

320 a significant age effect, $\mathrm{F}(2,49)=3.45, \mathrm{p}<0.05$; Bonferroni-corrected pair-wise analyses showed

321 a marginally significant difference between the 4- and 12-month-olds, with the 4-month-olds

322 having a smaller amplitude $(\mathrm{p}=0.06)$.

323

\begin{tabular}{|c|c|c|c|}
\hline & $1^{\text {st }}$ note & $2^{\text {nd }}$ note & $3^{\text {rd }}$ note \\
\hline $4 \mathrm{~m} \quad \mathrm{P} 1$ & $4.09(2.23)$ & $3.37(2.56)$ & $2.77(2.18)$ \\
\hline N2 & $1.96(2.34)$ & $1.50(1.86)$ & $1.81(1.99)$ \\
\hline $8 \mathrm{~m} \quad \mathrm{P1}$ & $3.26(2.36)$ & $1.99(2.81)$ & 2.78 (1.99) \\
\hline $\mathrm{N} 2$ & $1.76(2.15)$ & $0.51(2.09)$ & $1.00(1.76)$ \\
\hline $12 \mathrm{~m} \mathrm{P} 1$ & $3.53(1.45)$ & $2.59(1.67)$ & $2.70(2.07)$ \\
\hline $\mathrm{N} 2$ & $1.20(1.47)$ & $0.09(1.72)$ & $0.81(2.71)$ \\
\hline
\end{tabular}

324 Table 3. Mean P1 and N2 amplitude (in $\mu \mathrm{V}$ ) of each note of each age group. Standard deviations 325 are given in parenthesis.
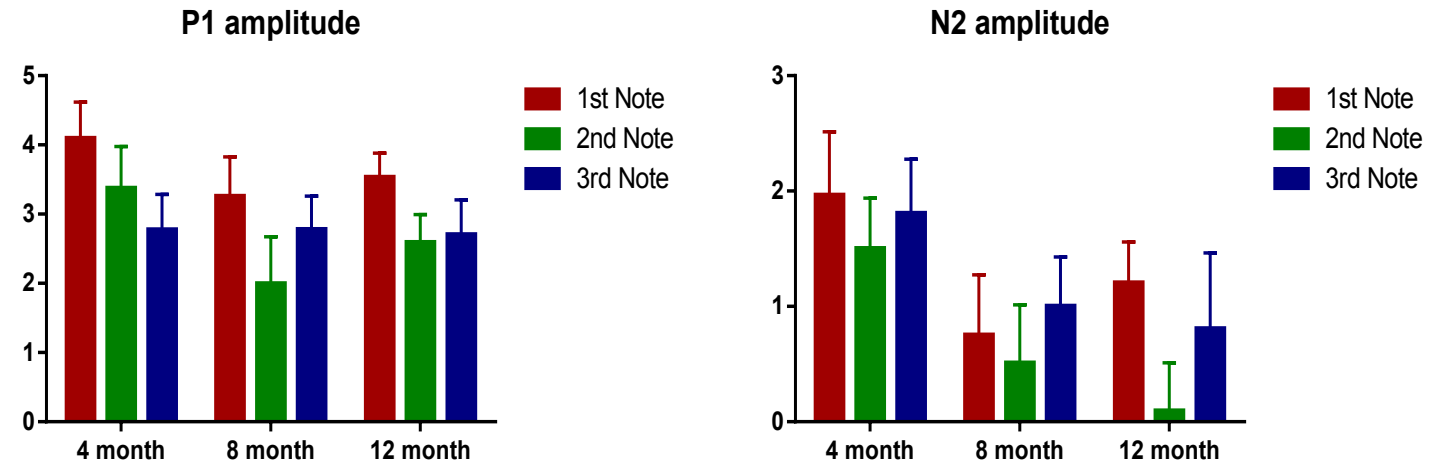

326 
327 Figure 4. Mean P1 and N2 amplitude of each note of each age group. Error bars represent

328 standard errors.

329 Discussion

330 In the present study, we tested 4-, 8- and 12-month-old infants on their auditory ERPs to

331 a three-note melody. For all the three successive notes, there was an overall decrease in P1 and

332 N2 latency over age. This indicates an increase in the speed of neural transmission, which is

333 presumably related to the increased myelination of neurons over age. Latency decrease has been

334 previously found in the response to single acoustic stimuli (e.g. Barnet et al., 1975; Novak et al.,

335 1989; Kushnerenko et al., 2002), but as the results here show decreasing latencies to successive

336 stimuli in a stream, this implies that infants are able to segment a sound stream into its composite

337 elements, with individual registration of each element at the neural level. Even at 4 months,

338 although the N2 was not fully expressed when the next note occurred, P1 for the next note was

339 still visibly evident. Nevertheless, as the onset response to the late events in a stream may

340 overlap with the response from preceding events, the non-initial response is possibly more

341 variable across participants. Kushnerenko et al. (2002) found a decrease in P1 but not in N2

342 latency from 6 to 12 months, and N2 was not visibly evident before 6 months. Little et al. (1999)

343 found a decrease in N2 but not in P1 from birth to 17 weeks. In the present study, we found

344 consistent latency decrease across age for both peaks. These different findings are possibly due

345 to the different stimuli: Little et al. (1999) used one single harmonic tone, Kushnerenko et al.

346 (2002) used three single harmonic tones, and we used a stream containing three piano tones. It is 
347 possible that the relatively rich spectral content of our stimuli allowed N2 to be visibly evident at

348 a younger age than in the Kushnerenko et al. (2002) study.

Interestingly, the middle Note 2 elicited an earlier P1 latency compared to the edge notes,

350

1 and 3. The early P1 of the middle note is unlikely to be solely due to the incomplete response

351 to the first note. If this were the case, then Note 3 should have had an equally early P1 peak. It

352 seems that infant brain does not respond to the acoustic events at different positions of a stream

353 in an identical manner. For the moment, it is difficult to ascertain what might cause the change in

354 P1 latency across successive notes, yet it seems that the response to sounds at a central position

355 in a stream tend to be more variable. In addition, for the 8-month-olds, the amplitude of the

356 middle note seems to be more attenuated compared to the response to the first and third notes.

357 Whether the neuronal processes are different for edge events and for medial events in a stream

358 should be further tested, preferably with streams containing more than three notes. The majority

359 words in English have a strong-weak pattern (Cutler \& Carter, 1987), and whether such metrical

360 structure might lead to a more prominent response to the onset event in an acoustic stream should

361 be investigated by testing participants whose native language exhibits the opposite metrical

362 pattern (e.g., Turkish).

363 With regard to amplitude, P1 was larger for the first than for the subsequent notes.

364 Neither P1 nor N2 amplitude changed significantly across age, although it should be

365 acknowledged that individual variation is large for amplitude measurements, and such variation

366 may have masked any age group differences. Even though the statistical tests failed to show any 
367 consistent age effect, the grand average waveforms are qualitatively different for each age (see

368 Figure 2). For the 4-month-olds, clear P1s and N2s can be observed for Note 1 whereas those to

369 the two subsequent notes are much more attenuated. For the 8-month-olds, compared to the edge

370 notes, the central Note 2 has smaller P1 and N2 amplitudes. In addition, the P1 peak of the

371 central note shows a plateau-like morphology, whereas the responses to the edge notes have

372 sharper P1 peaks. The P1 and N2 peaks of the 12-month-olds show a comparably equivalent

373 amplitude and peak width across all three notes. Thus in summary, compared to older 8- and 12-

374 month-old infants, the younger 4-month-old infants had a smaller P1-N2 deflection for Note 3,

375 even this was the final note, with no other acoustic event following it. This suggests that young

376 infants may have difficulties in responding to late occurring events in a stream. Such attenuated

377 amplitude may be due to neural refractoriness (Budd, Barry, Gordon, Rennie, \& Michie, 1998;

378 Gilley, Sharma, Dorman, \& Martin, 2005; Ritter, Vaughan, \& Costa, 1968; Sable, Low, Maclin,

379 Fabiani, \& Gratton, 2004; Sussman, Steinschneider, Gumenyuk, Grushko, \& Lawson, 2008) with

380 processing of late events being hindered by unfinished processing of preceding events. In other

381 words, more time may be required for neurons to reset and respond to following acoustic events

382 for young infants.

383 Conclusion

384 In this study we examined auditory cortical responses to successive musical notes of

385 infant at ages of 4, 8, and 12 months. Clear P1 and N2 peaks were identified for all three notes at

386 all three ages, indicating successful neural registration of individual components in a continuous 
387 stream. While there was a significant decrease in P1 and N2 latencies all three notes as infant age

388 increased, these results show, for the first time, individual neural registration of components of

389 continuous multi-element events, even in relatively young infants. Over and above this general

390 finding age-related nuances were evident: P1 and N2 amplitudes were larger for the first note in

391 the stream than for subsequent notes, and additionally, for the final Note 3, the 4-month-olds had

392 a smaller amplitude compared to the two older groups, suggesting that, despite individual

393 registration, young infants may have greater difficulty registering late occurring events in a

394 stream. This attenuated amplitude for late events at the younger age points to the possible nature

395 of developmental progression in auditory event registration in infancy; there could well be neural

396 registration of individual elements in an acoustic stream, but, possibly due to incomplete

397 processing of each event, there may be cumulative reduction of processing resources within a

398 certain refractory period. This would imply temporal and numerical limits on successive

399 component event processing, which are possibly related to memory limits. Further studies of

400 such limits and their reduction over age will greatly improve the understanding of speech

401 perception and language development as well as the development of other complex abilities such

402 as music and other event processing. For young infants, P1 and N2 latencies change over the

403 course of the experiment. This study provides the basis for such future studies by showing, for

404 the first time, that as young as 4 months, infants show specific neural registration of successive

405 frequency-modulated events in an otherwise continuous stream, and continuing maturation of the

406 brain response to successive sounds in the first year of life. 
408

409

410

411

412

413

414

415

416

417

418

419

420

421

422

423

424

425

426

Barnet, A. B., Ohlrich, E. S., Weiss, I. P., \& Shanks, B. (1975). Auditory evoked potentials during sleep in normal children from ten days to three years of age. Electroencephalography and Clinical Neurophysiology, 39(1), 29-41. doi:10.1016/0013-4694(75)90124-8

Budd, T. W., Barry, R. J., Gordon, E., Rennie, C., \& Michie, P. T. (1998). Decrement of the N1 auditory event-related potential with stimulus repetition: habituation vs. refractoriness. International Journal of Psychophysiology, 31(1), 51-68. doi:10.1016/S0167$\underline{8760(98) 00040-3}$

Ceponiene, R., Cheour, M., \& Näätänen, R. (1998). Interstimulus interval and auditory eventrelated potentials in children: Evidence for multiple generators. Electroencephalography and Clinical Neurophysiology - Evoked Potentials, 108(4), 345-354. doi:10.1016/S01685597(97)00081-6

Choudhury, N., \& Benasich, A. A. (2011). Maturation of auditory evoked potentials from 6 to 48 months: Prediction to 3 and 4 year language and cognitive abilities. Clinical Neurophysiology, 122(2), 320-338. doi:10.1016/j.clinph.2010.05.035

Cutler, A., \& Carter, D. M. (1987). The predominance of strong initial syllables in the English vocabulary. Computer Speech \& Language, 2(3-4), 133-142. doi:10.1016/0885$\underline{2308(87) 90004-0}$

Dehaene-Lambertz, G., \& Baillet, S. (1998). A phonological representation in the infant brain. NeuroReport, 9(8), 1885-1888. doi: 10.1097/00001756-199806010-00040 
427 Gilley, P. M., Sharma, A., Dorman, M., \& Martin, K. (2005). Developmental changes in

428 refractoriness of the cortical auditory evoked potential. Clinical Neurophysiology, 116(3),

429 648-657. doi:10.1016/j.clinph.2004.09.009

430 Giraud, A., \& Poeppel, D. (2012). Cortical oscillations and speech processing: Emerging

431 computational principles and operations. Nature Neuroscience, 15(4), 511-517. Retrieved

432 from $\underline{10.1038 / \mathrm{nn} .3063}$

433 He, C., Hotson, L., \& Trainor, L. J. (2007). Mismatch responses to pitch changes in early infancy.

434 Journal of Cognitive Neuroscience, 19(5), 878-892. doi:10.1162/jocn.2007.19.5.878

435 Jing, H., \& Benasich, A. A. (2006). Brain responses to tonal changes in the first two years of life.

436 Brain and Development, 28(4), 247-256. doi:10.1016/j.braindev.2005.09.002

437 Kushnerenko, E., Ceponiene, R., Balan, P., Fellman, V., \& Näätänen, R. (2002). Maturation of

438 the auditory change detection response in infants: a longitudinal ERP study. Neuroreport,

439 13(15), 1843-1848. doi: $\underline{10.1097 / 00001756-200210280-00002}$

440 Little, V. M., Thomas, D. G., \& Letterman, M. R. (1999). Single-trial analyses of developmental

441 trends in infant auditory event-related potentials. Developmental Neuropsychology, 16(3),

442 455-478. doi:10.1207/S15326942DN1603_26

443 Moelants, D. (2002). Preferred tempo reconsidered. Proceedings of the 7th International

444 Conference on Music Perception and Cognition, pp. 580-583.

445 Näätänen, R. (1992). Attention and brain function. New Jersey: Erlbaum. 
446 Näätänen, R., Paavilainen, P., Rinne, T., \& Alho, K. (2007). The mismatch negativity (MMN) in

447 basic research of central auditory processing: A review. Clinical Neurophysiology, 118(12), 2544-2590. doi:10.1016/j.clinph.2007.04.026

449

450

451

452

453

454

455

456

457

458

459

460

461

462

463

464

Novak, G. P., Kurtzberg, D., Kreuzer, J. A., \& Vaughan Jr., H. G. (1989). Cortical responses to speech sounds and their formants in normal infants: maturational sequence and spatiotemporal analysis. Electroencephalography and Clinical Neurophysiology, 73(4), 295305. doi: $10.1016 / 0013-4694(89) 90108-9$

Paavilainen, P. (2013). The mismatch-negativity (MMN) component of the auditory eventrelated potential to violations of abstract regularities: A review. International Journal of Psychophysiology, 88(2), 109-123. doi:10.1016/j.ijpsycho.2013.03.015

Ponton, C. W., Eggermont, J. J., Kwong, B., \& Don, M. (2000). Maturation of human central auditory system activity: evidence from multi-channel evoked potentials. Clinical Neurophysiology, 111(2), 220-236. doi:10.1016/S1388-2457(99)00236-9

Ritter, W., Vaughan, H. G., \& Costa, L. D. (1968). Orienting and habituation to auditory stimuli: A study of short terms changes in average evoked responses. Electroencephalography and Clinical Neurophysiology, 25(6), 550-556. doi:10.1016/0013-4694(68)90234-4

Sable, J. J., Low, K. A., Maclin, E. L., Fabiani, M., \& Gratton, G. (2004). Latent inhibition mediates N1 attenuation to repeating sounds. Psychophysiology, 41(4), 636-642. doi:10.1111/j.1469-8986.2004.00192.x 
465 Shucard, D. W., Shucard, J. L., \& Thomas, D. G. (1987). Auditory event-related potentials in

466 waking infants and adults: a developmental perspective. Electroencephalography and

467 Clinical Neurophysiology, 68(4), 303-310. doi:10.1016/0168-5597(87)90051-7

468 Stefanics, G., Háden, G. P., Sziller, I., Balázs, L., Beke, A., \& Winkler, I. (2009). Newborn

469 infants process pitch intervals. Clinical Neurophysiology, 120(2), 304-308.

470 doi:10.1016/j.clinph.2008.11.020

471 Sussman, E., Steinschneider, M., Gumenyuk, V., Grushko, J., \& Lawson, K. (2008). The maturation of human evoked brain potentials to sounds presented at different stimulus rates. Hearing Research, 236(1-2), 61-79. doi:10.1016/j.heares.2007.12.001

474 Tallal, P. (2004). Improving language and literacy is a matter of time. Nature Reviews $475 \quad$ Neuroscience, 5(9), 721-728. doi: $10.1038 / \mathrm{nrn} 1499$

476 Telkemeyer, S., Rossi, S., Koch, S. P., Nierhaus, T., Steinbrink, J., Poeppel, D., .. .

477 Wartenburger, I. (2009). Sensitivity of newborn auditory cortex to the temporal structure of $478 \quad$ sounds. The Journal of Neuroscience, 29(47), 14726-14733.

479 doi:10.1523/JNEUROSCI.1246-09.2009

480 Tew, S., Fujioka, T., He, C., \& Trainor, L. (2009). Neural representation of transposed melody in 481 infants at 6 months of age. Annals of the New York Academy of Sciences, 1169(1), 287-290. doi:10.1111/j.1749-6632.2009.04845.x 
483 Trainor, L. J. (2012). Musical experience, plasticity, and maturation: issues in measuring 484 developmental change using EEG and MEG. Annals of the New York Academy of Sciences, $485 \quad$ 1252(1), 25-36. doi:10.1111/j.1749-6632.2012.06444.x

486 Weitzman, E. D., \& Graziani, L. J. (1968). Maturation and topography of the auditory evoked 487 response of the prematurely born infant. Developmental Psychobiology, 1(2), 79-89. 488 doi:10.1002/dev.420010203

489 Wunderlich, J. L., Cone-Wesson, B. K., \& Shepherd, R. (2006). Maturation of the cortical $490 \quad$ auditory evoked potential in infants and young children. Hearing Research, 212(1-2), 185491 202. doi:10.1016/j.heares.2005.11.010 